\title{
Autonomic dysfunction in SARS-COV-2 infection acute and long-term implications COVID-19 editor's page series
}

\author{
Richard C. Becker ${ }^{1}$ \\ Accepted: 26 July 2021 / Published online: 17 August 2021 \\ (c) The Author(s), under exclusive licence to Springer Science+Business Media, LLC, part of Springer Nature 2021
}

\begin{abstract}
The autonomic nervous system (ANS) is a complex network of nerves originating in the brain, brain stem, spinal cord, heart and extracardiac organs that regulates neural and physiological responses to internal and external environments and conditions. A common observation among patients with the 2019 Coronavirus $(\mathrm{CoV})$ (SARS-severe acute respiratory syndrome CoV-2) (SARS-CoV-2) or COVID-19 [CO for corona, VI for virus, D for disease and 19 for when the outbreak was first identified (31 December 2019)] in the acute and chronic phases of the disease is tachycardia, labile blood pressure, muscular fatigue and shortness of breath. Because abnormalities in the ANS can contribute to each of these symptoms, herein a review of autonomic dysfunction in SARS-COV-2 infection is provided to guide diagnostic testing, patient care and research initiatives.
\end{abstract}

\section{Graphic abstract}

The autonomic nervous system is a complex network of nerves originating in the brain, brain stem, spinal cord, heart and extracardiac organs that regulates neural and physiological responses to internal and external environments and conditions. A common collection of signs and symptoms among patients with the 2019 Coronavirus (CoV) (SARS-severe acute respiratory syndrome CoV-2) (SARS-CoV-2) or COVID-19 [CO for corona, VI for virus, D for disease and 19 for when the outbreak was first identified (31 December 2019)] is tachycardia, labile blood pressure, muscular fatigue and shortness of breath. Abnormalities in the autonomic nervous system (ANS) can contribute to each of these identifiers, potentially offering a unifying pathobiology for acute, subacute and the long-term sequelae of SARS-CoV-2 infection (PASC) and a target for intervention.

Keywords COVID-19 $\cdot$ Post-acute sequelae of SARS-CoV-2 infection (PASC) $\cdot$ Dysautonomia

\section{Highlights}

- COVID-19 is associated with several common symptoms in the acute phase that can linger during recovery.

- These include tachycardia, shortness of breath, fatigue and post-exercise exhaustion.

- Because each symptom can be traced to the autonomic nervous system and its dysfunction, a platform for investigation is clear.

Richard C. Becker

richard.becker@uc.edu

1 Heart, Lung and Vascular Institute, University of Cincinnati College of Medicine, 231 Albert Sabin Way, Cincinnati, OH 45267, USA

\section{Introduction}

The autonomic nervous system is a complex network of nerves originating in the brain, brain stem, spinal cord, heart and extracardiac organs that regulates neural and physiological responses to internal and external environments and conditions (reviewed in Goldberger) [1]. A common collection of signs and symptoms among patients with the 2019 Coronavirus $(\mathrm{CoV})$ (SARS-severe acute respiratory syndrome CoV-2) (SARS-CoV-2) or COVID-19 [CO for corona, VI for virus, $D$ for disease and 19 for when the outbreak was first identified (31 December 2019)] is tachycardia, labile blood pressure, muscular fatigue and shortness of breath. Abnormalities in the autonomic nervous system (ANS) can contribute to each of these identifiers, potentially offering a unifying pathobiology for acute, subacute and the long-term sequelae of SARS-CoV-2 infection (PASC) and a target for intervention. 


\section{Pre-existing conditions}

Abnormalities in the ANS not uncommonly precede COVID19 and contribute to its morbidity and mortality (reviewed in Del Rio) [2]. Systemic hypertension, heart failure, diabetes mellitus, obesity and chronic kidney disease are characterized by heightened sympathetic tone, increased risk of infection and poor outcomes. The reason(s) are multifactorial, but in addition to pre-existing conditions that impair vital organ reserve and normal adaptive physiological responses to stressors, the anti-inflammatory potential of vagal cholinergic efferent activation may be limited by pre-existing or acquired autonomic

Table 1 Causes of autonomic dysfunction

\section{Primary}

Pure pandysutonomia

Pure cholinergic dysautonomia

Multi-system atrophy (Shy-Drager syndrome)

Autonomic failure and Parkinson's disease

Familial amyloid neuropathy

Familial dysautonomia

Dopamine $\beta$-hydroxylase deficiency

Baroreflex failure

Neurocardiogenic SYNCOPE

Cardiac

Myocardial ischemia

Myocardial infarction

Cardiomyopathy

Infiltrating disorders

Amyloidosis

Hemochromatosis

Fabray's disease

Eosinophil

Secondary

Diabetes mellitus

Chronic advance kidney disease

Chronic liver disease

Alcohol-related

Thiamine deficiency

Amyloidosis

Neurologic

Spinal cord injury

Transverse myelitis

Guillain-Barré syndrome

Vasovagal syndrome

Carotid sinus-baroreceptor dysfunction

POTS

Reflex sympathetic dystrophy

Multiple sclerosis

Infection

Human immunodeficiency virus

Parasitic disease (e.g. Chagas disease) dysfunction. This translates to heightened cytokine synthesis and release, as well as impaired restraint to inflammation in the heart, lungs and brain [3] (Tables 1 and 2).

The parasympathetic nervous system has an important immunomodulating effect [4]. Because patients with COVID-19 in general and those with PASC often display signs, symptoms and serologic findings of autoimmune disease, an understanding of autonomic dysfunction may offer bidirectional benefit.

\section{Anatomy of the autonomic nervous system}

The ANS is a highly complex network with integrated centers in the cortex, amygdala, hippocampus, and cingulate gyrus (limbic system), brainstem, spinal cord, thorax and heart. The network acts in response to internal and external stimuli and conditions at a subconscious level.

\section{The heart}

The intrinsic cardiac nervous system consists of cardiac ganglia located within fat pads superior and posterior to the
Table 2 Acquired autonomic dysfunction 
Fig. 1 An overview of the autonomic nervous system to include control regions of the brain, efferent and afferent systems, sympathetic and parasympathetic systems and reflex areas within the brainstem, intrathoracic and extra-thoracic locations. $D R G$ dorsal root ganglion, ICNS intrinsic nervous system, $S G$ stellate ganglion. From [1]

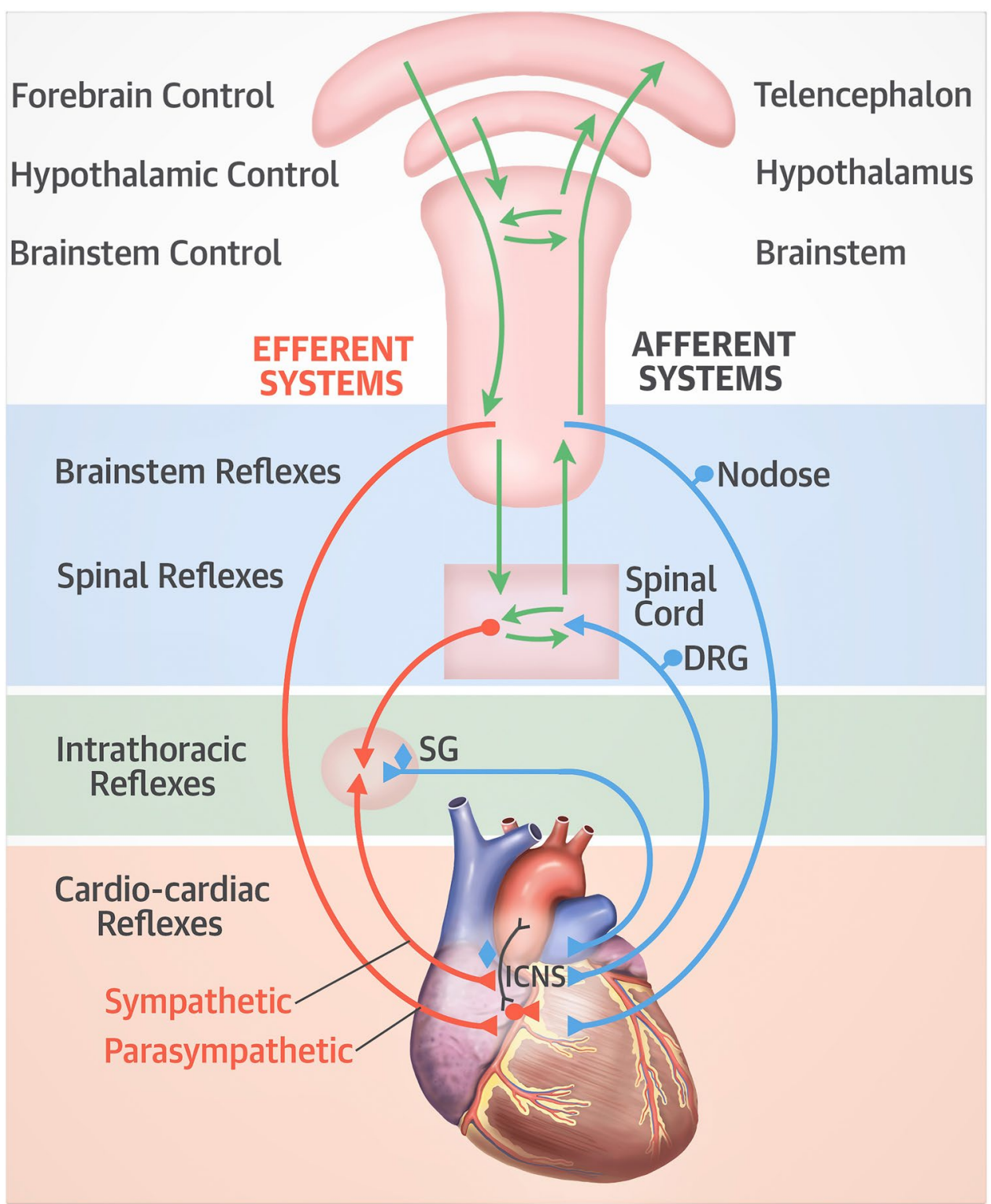

atria (reviewed in Armour) [5]. They are connected to the intrathoracic extracardiac ganglia, extra-thoracic cardiac ganglia (dorsal root ganglia) and the central nervous system. Afferent neural impulses originate within the heart and are transmitted to the intrinsic neurons of the heart, extrinsic or extracardiac intrathoracic ganglia (e.g. stellate ganglia), spinal cord and bran stem. Efferent neural impulses exist at each level through short and long feedback loops (Fig. 1). While a traditional description of the ANS includes the sympathetic and parasympathetic trunks, both contain afferent and efferent nerve fibers.

Armour et al. [5] performed microdissections and electron microscopy (EM) on six human hearts. Additional EM and histologic sections were obtained from the right atrial and medial ventricular ganglia of 12 other human hearts.
Collections of ganglia associated with nerves (i.e. ganglionated plexuses) were consistently found around the left and right atria as described previously, with fusion of posterior and medial plexuses and anterior extension to the interatrial septum. Ventricular ganglionic plexuses were consistently located within fat surrounding the aortic root, at the origins of the right and left coronary arteries with extension to the left anterior descending and left circumflex coronary arteries, at the origin of the posterior descending coronary artery and at the origins of the right and left marginal arteries (Fig. 2). The human heart is estimated to contain greater than 14,000 neurons.

The anatomic-physiological relationship that characterize the ANS is predicted, at least in part, by their geographical location and surrounding environment, including tissues and 
Fig. 2 Light photomicrographs of human intrinsic cardiac nerves, ganglia, and neurons. A Network of ganglia and nerves stained with methylene blue and dissected from the posteromedial left atrial ganglionated plexus. The ganglia appear as expansions along the length of a nerve, often at branch points (box). B Enlargement of boxed area in A illustrating a ganglion composed of approximately 150-200 nerve cell bodies. Note the presence of individual neurons in adjacent nerves. $\mathbf{C}$ High-magnification micrograph of a multipolar neuron. Neurons were usually round or ovoid in profile and frequently had dendritic processes extending into the center of the ganglion. Note the accumulation of lipofuscin granules (arrowheads). From [5]. With permission
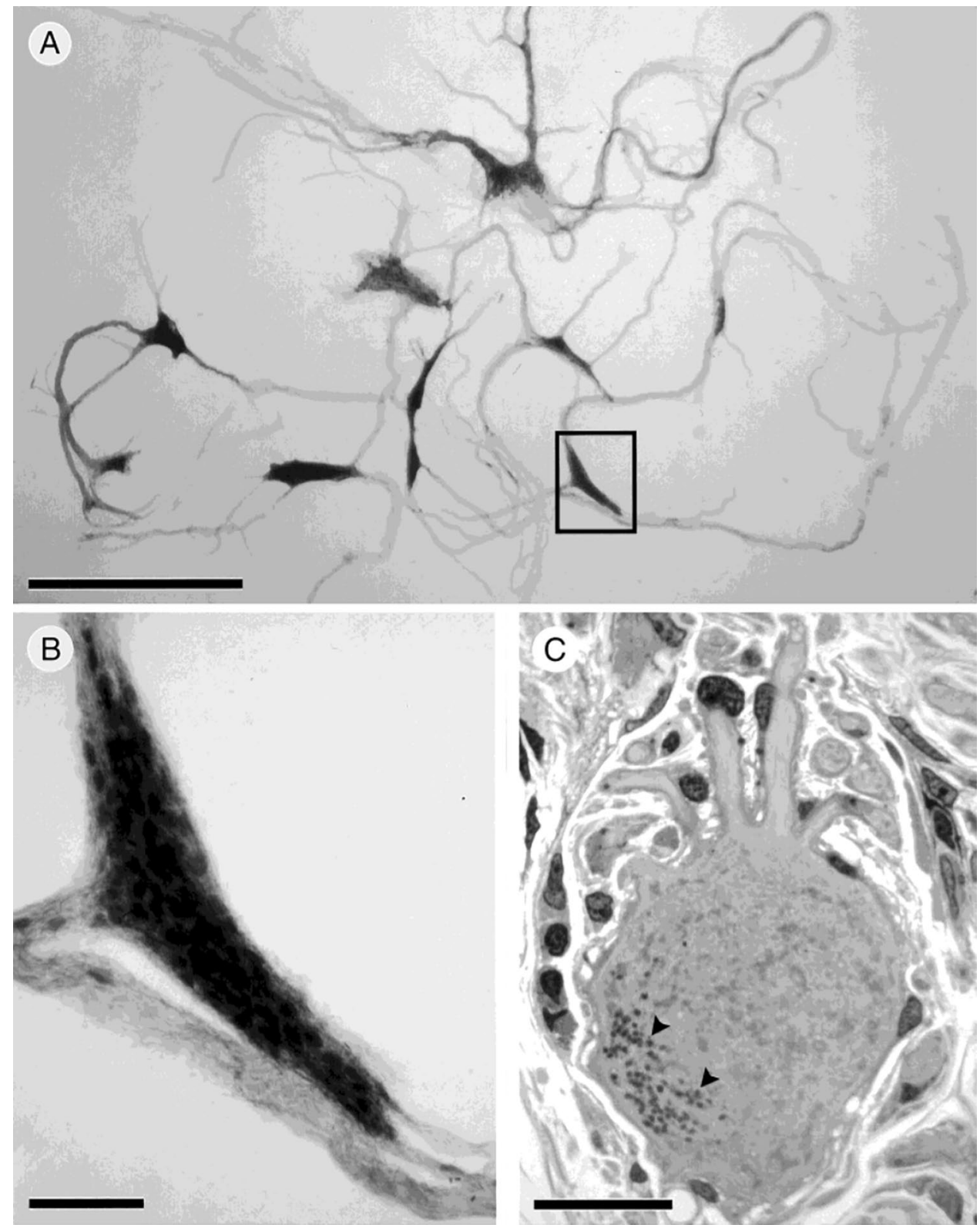

end-organs. Reflex loops are the functional underpinning for maintaining homeostasis under variable conditions and dynamic changes in response to environments encountered in health and disease (reviewed in Chouchou) [6].

\section{The brain}

As summarized previously, sympathetic activity follows a track or neural network in the rostral ventrolateral medulla, spinal cord and hypothalamus (paraventricular nucleus and lateral hypothalamus). Parasympathetic activity is directed by neurons in the nucleus ambiguus and dorsal motor nucleus of the vagus nerve. Both the sympathetic and parasympathetic systems receive vital information, either directly or indirectly through the solitary tract nucleus, from vasomotor and stretch sensitive afferent signaling from the lungs during ventilation (lung afferents), arterial pressure (aortic and carotid receptor) afferents, muscle receptor afferents, metabolic afferents, hypoxia and hypercapnia afferents and both somatic and visceral afferents [7].

\section{Central autonomic nervous system}

A traditional view of the ANS is based on peripheral responses to rapidly changing environments. A contemporary perspective highlights an equally important role of internal homeostasis as modulated by the central autonomic nervous system (reviewed in Reisert) [8]. Global 
tractography determined by diffusion-weighted imaging, positron emission tomography (PET) and functional magnetic resonance imaging (fMRI) performed on participants in the National Institutes of Health Human Connectome Project (launched in 2009 as a Blueprint Grand Challenge (www.nih.gov accessed May 1, 2021) have broadened our understanding of the ANS and its modulation substantially.

A detailed analysis of the cortical autonomic network performed by Reisert and colleagues [8] revealed the following: the cortical autonomic network includes three distinct pathways - the parietal-anterior-temporal, the occipital posterior-temporo-frontal and the limbic pathway contained within the amygdala, hippocampus, and cingulate gyrus.

\section{Physiology}

The intracardiac ANS coordinates sympathetic and parasympathetic input from other components of the extracardiac intrathoracic, extrathoracic, spinal and central ANS. The coordinating function allows regional control over several cardiac functions, including sinus node activation and propagation, as well as atrioventricular (AV) node conduction.

The sympathetic neurons that regulate cardiac performance are in the stellate ganglion. Efferent nerves course alongside of the coronary arteries and subsequently penetrate the epicardial surface of the heart, ending in the endocardium [1]. Stimulation of sympathetic efferent neurons increases inotropy, dromotropy, lusitropy and chronotropy. By contrast, cardiac afferent neurons are primarily chemosensory and mechanosensory [9], and participate in vasoreactivity, inflammatory responses and injury and cardiac remodeling. The parasympathetic afferent and efferent neurons participate in a variety of cardiac functions, including heart rate and blood pressure homeostasis, reduction and lowering, respectively.

The ANS regulates heart rate, cardiac output, blood pressure, respiratory rate and body temperature as well as other visceral activities. Sympathetic stimulation increases heart rate (chronotropy), contractility (inotropy) and AV conduction (dromotropy). It also causes vascular constriction, renin secretion and bronchiole dilation. By contrast, parasympathetic stimulation decreases heart rate, $\mathrm{AV}$ conduction and constricts bronchioles.

Under normal conditions sympathetic tone determines the amount of vasoconstriction and vasodilation of the peripheral vasculature. This is achieved through distinct responses to activation of $\beta$ and $\alpha$ adrenergic receptors. Sympathetic activation of $\beta_{1}$ receptors increases heart rate, AV conduction and ventricular contractility. $\beta_{2}$ activation of receptors located in the vascular smooth muscles, skeletal muscle and coronary arteries causes vasodilation and increases perfusion to the heart, vascular organs and skeletal muscles. $\alpha_{1}$ receptors are primarily expressed in the vascular smooth muscle cells and their activation causes vasoconstriction. $\alpha_{2}$ receptors are also expressed in vascular smooth muscle cells and respond to sympathetic signaling with vasoconstriction.

\section{The vasculature}

\section{Baroreceptors}

The ANS system maintains vital organ perfusion under varied conditions, including postural changes. Under normal circumstances, assuming an upright posture causes blood pooling in the lower extremities and splanchnic circulation. A rapid triggering of baroreceptors located in the carotid arteries and aortic arch increases sympathetic tone, vasoconstriction and an increased heart rate. Signaling of these vital homeostatic events is achieved by norepinephrine release from post-ganglionic sympathetic nerves (reviewed in Isaacson) [10]. Baroreceptor inflammation, injury and dysfunction have been postulated in the setting of COVID19 (reviewed in Becker) [11].

Baroreceptors are responsible for integrated functions between the heart and peripheral vessels. The arterial baroreceptor system is intimately involved with maintaining vascular tone and blood pressure homeostasis [12]. Arterial baroreceptors (stretch receptors located in the carotid sinuses and aortic arch) provide continuous feedback on blood pressure to the central nervous system, which responds with physiological efferent autonomic activity. Activation of arterial baroreceptors in response to increased blood pressure causes activation of vagal cardio-inhibitory neurons and a decrease of sympathetic neuron discharges to the heart and peripheral resistance bed [13]. The end-result is a decrease in heart rate, cardiac contractility, peripheral vascular resistance and venous return. By contrast, increased sympathetic activity and vagal inhibition leads to tachycardia and heightened cardiac contractility, vascular resistance and venous return.

Baroreflex activity is influenced by many factors, including respiratory, behavioral and environmental conditions (temperature, noise, light). Common cardiovascular diseases, ranging from coronary artery disease, myocardial infarction, essential hypertension and heart failure are associated with baroreceptor reflex abnormalities, primarily chronic adrenergic activation [14].

Whether or not COVID-19-associated vascular injury directly causes baroreceptor reflex abnormalities thereby contributing to autonomic dysfunction is a question under investigation. 


\section{Pericytes}

\section{Pericytes}

The vascular system includes endothelial cells, contractile smooth muscle cells and pericytes at the capillary level. There is continuous communication between pericytes and vascular endothelial cells that maintain vessel wall integrity, but also contribute to regulating inflammatory and immune processes (reviewed in Grönloh) [15]. There are chemical and physical interactions that include pericyte invagination, gap junction-based interactions, adherans junction-based interactions and cell-cell adhesion (reviewed in Dessalles) [16]. The physical interactions are believed to provide an opening or portal for leukocyte migration and extravagation from the circulation to the peri-vascular space. Given the wide variability between the number of pericytes per endothelial cell from one tissue to another, trans-endothelial migration of inflammatory cells might be tissue specific (reviewed in Shepro) [17].

The density of pericytes in each organ correlates with mechanical forces generated in the micro vessels. For example, there are a greater number of pericytes in the lower extremities compared to the upper extremities, the distribution of pericytes is greater in the brain and the kidneys because the vessels are exposed to greater pressure pulsatility, and inflammation-associated increased blood flow increases pericyte coverage over endothelial junctions [18]. The lungs of adults with pulmonary hypertension contain a twofold greater number of pericytes than non-hypertensive adults [19].

Gaps between pericytes can also serve as a site for leukocyte migration and are known to vary in size (width) and number according to local conditions. Inflammatory proteins such as tumor necrosis factor (TNF) and Interleukin (IL) $1 \beta$ increase the pericyte gap size and facilitate leukocyte migration [20].

Emerging evidence show pericyte heterogenicity and specific subsets that control blood-brain barrier permeability and vascular flow [21].

Pericytes wrap around endothelial cells and regulate several vascular functions, including inflammation and angiogenesis. They are also a major contributor of vascular tone. Quiescent pericytes are physically encased with the basement membrane and have two different substructures-the soma within the nucleus and elongated processed that either rack longitudinally along the vessel or wrap around the vessel circumference [22]. The tips of pericyte elongation penetrate through the basement membrane and join the endothelium in what is referred to as the maculae occludentes [22].

Activated pericytes appear to "breakout" of the basement membrane, elongated processes tract and detach from the vessel wall and migrate into parenchymal tissue. Pericyte activation occurs during angiogenesis and tissue regeneration, but it can also occur in response to stress-biochemical, mechanical or direct vascular injury. A sudden shift from a quiescent to an active state as commonly occurs with vessel injury causes pericytes to retract their processes (pseudopods), digest the basement membrane and migrate to surrounding tissues [23].

Litvinukova et al. [24] reported that SARS-CoV-2 ACE2 receptor transcripts are highest in pericytes.

Single-cell studies of SARS-CoV-2 entry genes [25] ACE2 and accessory proteases (TMPRSS2 and CTSL) have shown covariance with immune signaling genes. This is particularly evident with double positive $\left(\mathrm{ACE}^{+}{ }^{+}\right.$and TMPRSS $2^{+}$) cells in tissue programs enriched in pathways related to antigen processing and presentation: the expression of antigen-producing genes by infected cells is one of several potential mechanisms for autoimmune responses in VOCID-19 [25].

\section{The lungs}

The larynx, tracheobronchial tree, lung, pleura and diaphragm are innovated by an extensive network of sensory neurons (reviewed in Behrens) [26]. Different nerves monitor and respond to the physical micro-environment and chemical conditions of the airways. They also share pathways and sensory routes with cardiovascular afferent nerves within jugular vein vagal ganglia, dorsal root ganglia (extracardiac, intra-thoracic), brain stem, cortical and subcortical regions and the spinal cord [27].

Reactive airway disease is associated with autonomic imbalance. Specifically, a higher heart rate is observed in persons with asthma during exercise compared to agematched controls without asthma and a slower heart rate recovery [28]. Pulmonary vein isolation performed for refractory atrial fibrillation is associated with reduced heart rate variability and higher daytime and nighttime heart rate [29].

In humans, the sympathetic and parasympathetic nerves arising from the spinal ganglions and vagus nerve, respectively, merge at the carina to form the anterior and posterior plexi (reviewed in Garcia-Lunar) [30]. The nerves subsequently enter the lungs and form the peri-bronchial plexus and peri-arterial plexus. The latter courses all the way to the pulmonary microvasculature (arterioles $<100 \mu \mathrm{m}$ in diameter).

Autonomic dysregulation is observed commonly in persons with pulmonary hypertension of varied etiologies. While the initial trigger is likely multifactorial, pulmonary vascular injury and oxidative stress are likely involved [31]. The $\beta$-blocker nebivolol reduced endothelial cell proliferation and improved nitric oxide availability in a rat model of pulmonary hypertension [32]. Nebivolol has $\beta_{1}$ adrenergic 
receptor antagonist properties, but also possesses $\beta_{2}$ and $\beta_{3}$ receptor agonist vasodilating properties.

\section{Physiology}

Autonomic dysfunction is common among patients with COPD and results from involved chemoreflexes, decreased baroreceptor reflexes, rapid and slow breathing patterns and chronic activation of pulmonary stretch receptors [33]. Impaired exercise tolerance is multifactorial and related to cardiac sympathetic predominance, decreased response to both sympathetic and parasympathetic stimuli that alters cardiovascular and pulmonary function, muscle tone, and impaired exercise tolerance [34].

Airway sensory receptors channel information to the central nervous system that regulates breathing and other pulmonary function parameters. This degree of crosstalk is achieved by three distinct airway receptors. C-fiber receptors, rapidly adapting receptors, and slowly adapting receptors [35]. There is also deflation activated receptors (mechano-receptors).

The parasympathetic nervous system regulates pulmonary mucous production, airway smooth muscle tone, ciliary motility and transport, mucous secretion, cough reflexes and regulates local pulmonary inflammation and immunity.

\section{Generalized autonomic dysfunction}

Pure autonomic failure (PAF), also referred to as BradberryEggleston Syndrome is classified as a rare neurogenerative disease of the ANS. Typically, only the peripheral ANS is involved as a result of an abdominal accumulation of proteins (Lewy bodies) in the nerve cells (www.rarediseases. nih.gov; accessed May 29, 2021). Symptoms can begin at any age, but more typically in midlife and include orthostatic hypotension, dizziness, weakness, fatigue, constipation, sweating, neurogenic bladder and interrupted sleep. The differential diagnosis includes Parkinson's Disease, dopamine $\beta$-hydroxylate deficiency, amyloidosis, paraneoplastic syndromes, familial dysautonomia, autoimmune autonomic ganglionopathy, and multiple system atrophy. Treatment options include midodrine and droxidopa (indications-orthostatic hypotension in patient with Parkinson's Disease, multiple system atrophy, PAF, dopamine $\beta$-hydroxylase deficiency and non-diabetic autonomic neuropathy).

\section{Acute pan-dysautonomia}

Acute pan-dysautonomia is a rare condition characterized by severe sympathetic and parasympathetic impairment with relatively preserved sensory and motor function. There is loss of myelinated and unmyelinated fibers. Treatment includes parasympathomimetic or sympathomimetic agents $[36,37]$.

\section{Dyspnea and the ANS}

Breathlessness is a common symptom in patients with POTs and has been associated with dysfunctional breathing and hypoventilation syndrome [38].

Dyspnea in low pre-load states with accompanying reduced cardiac output may contribute to symptoms among patients with autonomic dysfunction [39].

Dynamic hyperinflation is common among patients with autonomic dysfunction and contributes to dyspnea in the absence of cardiovascular disease [40]. Patients with COPD are at particular risk and already have increased ventilatory workload and decreased inspiratory muscle capacity.

\section{Sleep}

Sleep and wakefulness are characterized by specific autonomic states, activity and physiological effects. For example, during non-rapid eye movement sleep (NREM), autonomic activity changes as does its interaction with endocrine, respiratory, cardiovascular and thermoregulatory systems (reviewed in Cerri) [41]. Considerable autonomic variation is observed in each stage of sleep (wakefulness, NREM sleep, and REM sleep) [42]. Accordingly, dysregulated sleep affects bodily homeostasis during wakefulness. Monti et al. [43] studied autonomic control of the cardiovascular system during sleep in 12 healthy adults. The greatest vagal activity was observed during NREM sleep, with the lowest sympathetic activity. Baroreflex sensitivity was greater during sleep than nocturnal wake periods and further increased during REM sleep.

Heart rate variability during sleep is also a measure of autonomic activity (reviewed in Choucou) [6]. There is higher parasympathetic tone during NREM sleep compared to the sympathetic hyperactivity that characterizes REM sleep.

\section{Exercise}

The ANS is intimately involved in exercise performance and endurance with both direct and indirect effects. Heart rate response to exercise and its recovery are altered in people with autonomic dysfunction, including when exercise is performed in the supine position [44]. An improvement in autonomic activity occurs with interval training and slow breathing exercise [45] in several conditions and disease states, including advanced age, prolonged periods of inactivity, chronic obstructive lung disease [46], obesity and following insufficient sleep [47]. 
Myalgic encephalomyelitis (CME) or chronic fatigue syndrome is characterized by impaired stamina and post-exercise exhaustion. Because antibodies against $\beta_{2}$ adrenergic receptors have been reported in $\mathrm{ME}$, autonomic dysfunction with vasoconstriction of blood vessels to the brain and exercising muscles and concomitant metabolically stimulated release of endogenous vasodilators causing suppression of renin and hypovolemia could represent a unifying pathophysiological process (reviewed in Wirth) [48]. Chronic fatigue is a common feature of PASC. Autonomic dysfunction in autoimmune disease may contribute to functional decline and heightened inflammation (reviewed in Stute) [49].

\section{Sighing}

Sighing is a normal response to autonomic activation and occurs on average 12 times per hour. The act of deep breathing or more specifically taking a deep breath before fully exhaling from a prior breath permits optimal alveolar gas exchange [50]. The physiological response to sighing is quite complex and includes a transient fall in central venous pressure (Phase 1) and period of apnea of varied duration (Phase 2).

The initial response to sighing as described above is followed by tachycardia and a rise in arterial blood pressure (Phase 1). The period of apnea includes concomitant bradycardia and reduced arterial blood pressure (Phase 2). During phase 2 , bronchial and coronary blood flow increase by 2-3-fold from baseline [51]. Post-sigh duration of expiration increases by several fold and delays the onset of subsequent inspiration. The observed pattern is related to a reduction in alveolar $\mathrm{PA} \mathrm{CO}_{2}$ and carotid chemoreceptor signaling [52].

Autonomic dysfunction with an increased number of hourly sighs, sigh rates and impaired vasoconstriction has been described in panic disorders [53] and dysfunctional breathing of varied etiologies (reviewed in Boulding) [54]. Many patients with PASC describe increased sighing throughout the day.

\section{Dysfunctional breathing}

Dysfunctional breathing is a broad term that describes a change in breathing patterns, impaired synchrony of diaphragmatic and respiratory muscle movement and thoracoabdominal asynchrony (reviewed in Boulding) [54] in the absence of significant respiratory or cardiac disease. A classification scheme for dysfunctional breathing proposed by Boulding and colleagues [54] can be summarized using the following categories: hyperventilation syndrome; periodic deep sighing with an irregular breathing pattern; thoracic dominant breathing and; forced abdominal respirations. The most common symptoms of dysfunctional breathing are dyspnea, tachycardia, impaired physical stamina and sighing with associated air hunger [55]. This collection of signs and symptoms is very common in PASC.

Debeaumont et al. [56] performed cardiopulmonary exercise tests (CPET) on 23 patients with persisting dyspnea 6 months after their initial diagnosis of COVID-19. $\mathrm{VO}_{2}$ peak was reduced to either a mild or moderate degree and, in all patients, respiratory equivalents were high, power-toweight ratios were low and ventilatory efficiencies were low (mean VE/ $/ \mathrm{VCO}_{2}$ slope) The findings suggest that persistent dyspnea was the cumulative result of muscle deconditioning and dysfunctional breathing.

\section{The autonomic nervous system in COVID-19}

Bajic et al. identified features of cardiac autonomic dysfunction in patients with COVID-19 employing entropy analysis applied to integrated transformed probability signals, common symbolic dynamics entropy and copulaparameters [57]. Interrupted sleep and irregular sleep patterns are common in patients with COVID-19. Either can cause impaired autonomic tone and endothelial vasomotor dysfunction [47] (Tables 3 and 4).

Large registries, including social media sites that include detailed questionnaires (Body Politic) have identified frequent sighing as a common symptom among patients with COVID-19 (wearebodypolitic.com accessed June 12, 2021). Tilt table testing is frequently abnormal in persons with unexplained sighing, suggesting a relationship with autonomic dysfunction [58].

Denis et al. [59] employed the COVID symptoms study database, a smart phone tracker database of 2.8 million recorded users to determine long-term symptoms of patients with COVID-19. They have encountered patients with orthostatic intolerance syndromes with symptoms of either hypotension at rest or upon standing and/or tachycardia. All their patients have been women between the ages of 26 and 50 years. Other investigators have utilized wearable technology to track long-term symptoms [60, 61].

Johansson and colleagues described a small case series of patients with POTS-like syndrome 3 months after a diagnosis of COVID-19 [62]. Each patient met the diagnostic criteria (table) and symptoms developed after a period of post-acute illness quiescence.

There have been cases of demyelinating diseases believed to be from COVID-19 [63] with accompanying sensory and motor deficits, ataxia and autonomic dysfunction. Involvement of the brain and spinal cord have been reported, including long extensive transverse myelitis [64].

Guillain-Barré Syndrome can be associated with profound autonomic dysfunction [65]. Occasionally, COVID-19 
related dysautonomia occurs along with cranial polyneuritis, neurosensory hearing loss and/or neuroophthalmological disorders [66]. The neurological sequelae associated with COVID-19 span a broad range from headaches and encephalopathy to encephalitis, meningitis, seizures, ischemic and hemorrhagic strokes, dural venous sinus thrombosis, Guillain-Barré Syndrome, peripheral neuropathy (cranial nerve, sensory, motor) and the focus of the current review, autonomic dysfunction. A question raised early in the pandemic was whether the central, peripheral and autonomic involvement was from direct virus invasion, immune-mediated, hypoxia, or a combination of toxic events.

In patients with COVID-19 and underlying COPD, there is a relationship between declining airflow, dynamic pulmonary hyperinflation and diffusion capacity with autonomic dysfunction and circulating markers of inflammation [33].

Patients with POTs often have a broad range of symptoms that collectively support the potential association of autonomic dysfunction in COVID-19 with PASC. The symptoms include palpitations, lightheadedness, breathlessness, chest discomfort, neuropathic pain, chronic fatigue, impaired/ interrupted sleep, gastrointestinal symptoms, cognitive slowing and anxiety [67].

\section{Postural orthostatic tachycardia syndrome}

The hallmark of classic POTs is orthostatic symptoms and tachycardia in the absence of significant hypotension (Table 5). Sympathetic excitation rather than a decrease in mean arterial pressure is believed to be responsible for

Table 3 Symptoms of autonomic dysfunction

Dizziness, lightheadedness, vertigo, syncope
Resting tachycardia
Palpitations
Chest pain
Altered bowel habits (constipation, diarrhea)
Nausea
Shortness of breath
Mood swings
Fatigue
Impaired stamina
Post-exercise exhaustion
Tremors
Interrupted sleep patterns
Sweating
Impaired concentration
Anxiety
Sound and light sensitivity
Headaches

Dizziness, lightheadedness, vertigo, syncope

Resting tachycardia

Palpitations

Chest pain

Altered bowel habits (constipation, diarrhea)

Nausea

Shortness of breath

Mood swings

Fatigue

Impaired stamina

Post-exercise exhaustion

Tremors

Interrupted sleep pattern

Sweating

Impaired concentration

Sound and light sensitivity

Headaches
Table 4 Triggers of symptoms in persons with autonomic dysfunction

Hot and humid environments

Dehydration

Alcohol consumption

Psychological stress

Sleep deprivation

Prolonged standing

Tight clothing

Over exertion

the symptoms, however, hypovolemia can cause a marked increase of symptoms, including syncope. This can be observed clinically and while a transient decrease in systolic blood pressure of $\geq 20 \mathrm{mmHg}$ is common during tilt testing, recovery typically occurs within one minute. A hyperadrenergic state with increased plasma norepinephrine levels helps to maintain blood pressure while standing. Deconditioning and loss of adrenergic tone can worsen existing symptoms. Low-dose $\beta$-blockers can reduce heart rate and attenuate symptoms in some, but not all patients [68].

\section{Inappropriate sinus tachycardia}

Inappropriate sinus tachycardia is associated with high heart rates at rest ( $>100$ beats per minute) and with even modest physical activity [69]. Heightened sympathetic tone and adrenergic activity are believed to be responsible, however, sick sinus syndrome may be a contributing factor in some patients (Table 6). In addition, some patients have IgG anti- $\beta$ receptor antibodies. Other potential contributors include $\beta$-receptor super sensitivity, impaired baroreflex control, blunted response to adenosine, central autonomic stimulation, impaired parasympathetic control and a gain-of-function channelopathy involving $\mathrm{I}_{\mathrm{f}}$ channels [70].

The management of patients with inappropriate sinus tachycardia includes excluding underlying, precipitating or contributing causes, graded exercise programs, $\beta$-blockers (nadolol, ivabradine or their combination), calcium channel blockers (verapamil) and partial sinus node ablation [71-73].

\section{Mechanisms of autonomic dysfunction in COVID-19}

Hematogenous spread of SARS-CoV-2, including across the blood-brain barrier, via trans-synaptic entry of infected neurons (retrograde dissemination), through olfactory nerves and occular epithelium has been scribed (reviewed in Engin) [74]. Neuronal entry of SARS-CoV-2 is believed to evade immune surveillance by basigin and neuropilin-1 receptors (Ulrich). In addition, cytokine surges impair the blood-brain vascular plexus, facilitating virus entry [75].

SARS-CoV-2 could enter the brain via macrophages that cross the blood-brain barrier [76], Plasma biomarkers of 
Table 5 Typical clinical presentation of POTS

Cardiovascular symptoms (pathognomonic)

Cardiovascular system

Noncardiovascular symptoms

General symptoms

Nervous system

Musculoskeletal system

Gastrointestinal system

Respiratory system

Urogenital system

Skin
Main: orthostatic intolerance, orthostatic tachycardia, palpitations, dizziness, lightheadedness, (pre-)syncope, exercise intolerance

Other frequent symptoms: dyspnea, chest pain/discomfort, acrocyanosis, Raynaud phenomenon, venous pooling, limb edema
General deconditioning, chronic fatigue, exhaustion, heat intolerance, fever, debility

Headache/migraine, mental clouding ("brain fog"), cognitive impairment, concentration problems, anxiety, tremulousness, light and sound sensitivity, blurred/tunnel vision, neuropathic pain (regional), sleeping disorders, involuntary movements

Muscle fatigue, weakness, muscle pain, fasciculations

Nausea, dysmotility, gastroparesis, constipation, diarrhea, abdominal pain, weight loss

Hyperventilation, wheezing, shortness of breath

Bladder dysfunction, nocturia, polyuria

Petechiae, rashes, erythema, telangiectasias, abnormal sudomotor regulation, diaphoresis, pallor, flushing

Adapted from Fedorowski (67)

POTS postural orthostatic tachycardia syndrome

CNS injury-neurofilament light chain protein (a maker of intra-axonal neuronal injury) and glial fibrillary acidic protein (a maker of astrocyte activation and injury) are elevated in patients with moderate-sever COVID-19 [77].

Neural and glial cells express angiotensin convertingenzyme (ACE)-2 receptors. The primary binding site for SARS-CoV-2 RNA virus has been detected in brain neurons and cerebrospinal fluid [78] in patients with COVID-19.

SARS-CoV-2 neurotropism can provide an explanation for COVID-19 dysautonomia in several ways. Infection of the olfactory neuroepithelium provide direct access to several regulatory centers of the brain by first traveling across nasal dendrites, the olfactory bulb (axonal retrograde transport) via the cribriform plate, and trans-synthetic transfer to the amygdala, hypothalamus, limbic and pre-frontal cortex, piriform cortex and brainstem.

SARS-CoV-2 neurotropism can provide an explanation for COVID-19 dysautonomia in several ways. Infection of the olfactory neuroepithelium provide direct access to several regulatory centers of the brain by first traveling across nasal dendrites, the olfactory bulb (axonal retrograde transport) via the cribriform plate, and trans-synaptic transfer to the amygdala, hypothalamus, limbic and pre-frontal cortex, piriform cortex and brainstem [78] - a primary center for cardiovascular and pulmonary autonomic regulation.

The neurological symptoms described previously could be from microvascular thrombosis-itself the result of endothelial cell invasion, inflammation and injury (reviewed in Becker) [79], immune-induced injury and activation of tissue factor or a combination $[80,81]$.
The systemic and localized inflammatory state that characterizes COVID-19 is associated with decreased monoamines, trophic factors and activation of microglia (reviewed in Boldini) [82]. SARS-CoV-2 could enter the brain through circumventricular organs, midline structures near the third and fourth ventricles and/or the area postrema [80].

\section{Duration of dysautonomia}

While the heterogeneity of COVID-19 and its phenotypes creates varying post-acute sequelae courses, several investigators have reported dysautonomia persisting for nearly a year following initial infection [83]. At times accompanied by symptoms severe enough to preclude a return to work.

\section{Autonomic dysfunction in COVID-19: diagnosis and management}

\section{Normal autonomic responses}

The autonomic dysfunction observed in PASC does not represent complete failure in most cases (Table 7). An exception might be in patients with G-B Syndrome in whom severe, pandysautonomia can occur [84].

\section{Autonomic testing}

A thorough assessment of the ANS includes an autonomic reflex screen of post-ganglionic sympathetic sudomotor function, cardiovagal function, cardiovascular adrenergic 
Table 6 Causes of sinus tachycardia

\section{Stimulants}

Caffeine

Nicotine

Alcohol

Amphetamines

Meth-amphetamines

Cocaine

Theobromines

\section{Cardiovascular}

Hypertension

Reduced parasympathetic tone

Heart failure

POTS (postural orthostatic tachycardia syndrome)
Cardiogenic Shock

Myocardial Infarction

Stroke

Pulmonary Embolism

Intracranial Hemorrhage

\section{Infection}

Sepsis

Central Nervous System

\section{Endocrine}

Hypertension

Adrenal insufficiency

Pheochromocytoma

Carcinoid Syndrome

Hyperglycemia

\section{General}

\begin{tabular}{|l|l|}
\hline Anxiety & Exercise \\
Fever & Hypoxia \\
Anemia & Pneumothorax \\
Psychosis & Dehydration \\
& Pregnancy \\
\hline
\end{tabular}

\begin{tabular}{l} 
Panic Attacks \\
Manic states \\
Malignant Hyperthermia \\
Allergic \\
reaction/anaphylaxis \\
Trauma \\
\hline
\end{tabular}

\section{Medication}

Clonidine Withdrawal

B-blocker withdrawal

Epinephrine

Dopamine

Norepinephrine

Theophylline

$\beta 1$-agonists 
Table 7 Diagnostic criteria of POTS

Sustained heart rate increment of not less than 30 beats/min or above 120 beats/min within 10 min of active standing or head-up tilt. For individuals who are younger than 19 years of age the required increment is at least 40 beats $/ \mathrm{min}$

Absence of orthostatic hypotension (i.e., sustained systolic blood pressure drop of not less than $20 \mathrm{mmHg}$ )

Reproduction of spontaneous symptoms such as light-headedness, palpitations, tremulousness, generalized weakness, blurred vision, and fatigue. In some patients, tachycardia may evoke vasovagal syncope corresponding to spontaneous attacks from patient's history

History of chronic orthostatic intolerance and other typical POTS-associated symptoms (for at least 6 months)

Absence of other conditions provoking sinus tachycardia such as anxiety disorders, hyperventilation, anemia, fever, pain, infection, dehydration, hyperthyroidism, pheochromocytoma, use of cardioactive drugs (sympathomimetics, anticholinergics)

Adapted from Johansson et al. [62]s

POTS Postural orthostatic tachycardia syndrome

function and a thermoregulatory sweat test (Low, Shouman). In addition to Tilt Table Testing, Valsalva maneuver, deep breathing test, isometric exercise test and heart rate variability, either in a dedicated laboratory or assessed with a 24-h Holter monitor with appropriate software are mainstays for assessment. Heart rate variability has also been determined in hospitalized patients with COVID-19 using an abbreviated 5-min protocol [85]. Measurement of plasma catecholamines may be useful (reviewed in Guaraldi) [86].

Heart rate variability has been used for decades as a surrogate marker for parasympathetic tone [87] in patients with cardiovascular and inflammatory diseases. While more recent observations support an interplay between parasympathetic and sympathetic tone, heart rate variability does inversely relate to markers of inflammation in patients with coronary artery disease, cardiovascular risk factors, autoimmune disease and traumatic brain injury $[88,89]$.

Non-invasive neuromodulation has been proposed for patients with COVID-19 and its long-term sequelae [90]. The goal of treatment is to reduce inflammatory medications and lessen the wide range of symptoms that could be autonomic in nature.

\section{Autoimmune dysfunction: management}

The first step in management is helping patients understand what they are experiencing and its basis in the context of post-acute sequelae. Adequate hydration and liberal sodium intake are important with 3-4 quarts daily-alternating water and fluids supplemented with electrolytes (e.g.) and two teaspoons of salt, respectively. It is particularly important to instruct patients on slow changes in posture, particularly from sitting to standing that should be done in stages (sitting for several minutes and standing for several minutes/ before walking).

Respiratory therapy and breathing retraining can improve functional capacity, respiratory rate, breath hold time and quality of life among patients with autonomic dysfunction, including those with POTs [38].
Treatment of dysfunctional breathing can be approached using several breathing techniques (Reviewed in Boulding) [54]. Two common techniques include the Papworth and Buteyko methods. The Papworth method centers on diaphragmatic breathing with an emphasis on controlled, slow nasal breathing. The Buteyko method employs nasal breathing with controlled pauses to limit periods of hyperventilation.

Autonomic dysfunction that causes supine hypertension and orthostatic hypotension is particularly challenging to manage, however, data are available to guide clinicians, the goal is to mitigate the end-organ injurious effects of nocturnal hypertension, while not worsening orthostatic hypertension and its potential risk of syncope, traumatic injury and poor quality of life [91].

Close monitoring of heart rate, blood pressure, medication and dose selection and symptoms is the cornerstone of optimal management. Most patients will not tolerate preload reducing agents like diuretics, nitrates and vasodilators. Eplerenone $50 \mathrm{mg}$ administered in the evening has been shown to lower nocturnal blood pressure, without worsening orthostatic hypotension. The benefit suggests that excess mineralocorticoid secretion may be a contributing mechanism of nocturnal hypertension [92]. Nebivolol has been used successfully [93] as has a low-dose nitroglycerine patch $(0.1 \mathrm{mg} / \mathrm{h})$ administered at night and removed in the morning [94]. Midodrine, a peripherally selective $\alpha$-1-adrenogic agonist increases vasomotor tone and peripheral vascular resistance and may limit the symptoms of orthostatic hypotension. Pyridostigmine, an anti-cholinesterase inhibitor has sympathomimetic properties and may limit orthostatic changes in blood pressure. Fludrocortisone can be used to expand intravascular volume if hydration (at least 2-3 L per day) and liberal sodium intake ( $4 \mathrm{~g}$ per day) are not sufficient.

Hyperadrenergic symptoms resulting from catecholamine surges with activity are common in PASC. The heightened sympathetic state can be particularly troubling for patients and impacts both stamina and quality of life. $\beta$-adrenergic 
blocker may limit the extent of heart rate rises. While clonidine and methyldopa can reduce sympathetic drive, many patients do not have a sufficiently high bold pressure to tolerate their use.

Supine hypertension occurs in many patients with autonomic dysfunction and failure (Okamoto) and can cause symptoms, end-organ injury and worsen day-time orthostatic hypotension from diuresis and volume depletion. Okamoto et al. [93] showed that passive heat (heating pad applied to the abdomen) reduced systemic blood pressure by $\sim 20 \mathrm{mmHg}(\mathrm{n}=12)$. In a proof-of-concept study performed in patients with autonomic failure, overnight passive heat $\left(38{ }^{\circ} \mathrm{C}\right.$ applied with a water perfused heating pad placed under the back) reduced systolic blood pressure by $28 \pm$ $6 \mathrm{mmHg}$.

Ampreloxetine, a selective, long-acting norepinephrine reuptake inhibitor has been studied in patients with symptomatic neurogenic orthostatic hypotension [95]. In a phase 2 clinical trial, escalating doses of ampreloxetine given over 5 days, followed by 20 weeks of open-label treatment, plasma norepinephrine levels increased by $71 \%$. Additional clinical trials with clinical end points are needed.

\section{Education}

We provide teaching materials to all patients attending the COVID-19 clinic. There are online sites that also provide important information for living a safe and content life (Autonomic Disorders Consortium; www.dysautonomiaint ernational.org).

\section{Things to avoid}

We encourage our patients to avoid standing for long periods of time, dehydration, alcohol, and upright exercise (if there are limiting symptoms). Recumbent exercise bikes, rowing, arm ergometers and swimming are favored.

We also instruct patients on sitting exercises that include resistance or counter pressure measures with $5 \mathrm{~s}$ of muscle tension followed by relaxation of the calfs, thighs, buttocks, abdomen, and arms, typically by crossing one over the other. These exercises increase venous return and blood pressure by primary vasculature for postural changes. Support stockings, or measured compression stockings (knee, thigh or belt/waist high as needed) are particularly important and should be used during the day and removed at night. We typically begin with $8-15 \mathrm{mmHg}$ and reserve $15-20$ or 20-30 $\mathrm{mmHg}$ for patients with pre-COVID orthostatic intolerance syndromes, varicose veins or concomitant peripheral edema. The stockings should be snug, but not painful.

Treatment with mineralocorticoids (e.g. fludrocortisone) or midodrine, an $\alpha$-receptor agonist that increases venule and arteriolar tone are reserved for patients with severe and persisting symptoms despite following all other recommendations as previously described. If advanced treatment is required and particularly if symptoms persist, a thorough evaluation for other causes of orthostatic intolerance should be undertaken to exclude life-altering and life-threatening conditions.

\section{Transcranial magnetic stimulation}

There are several areas of the brain that contribute to parasympathetic tone via the vagus nerve. The dorsal left prefrontal cortex (DL PFC), pregenual and mid-anterior cingulate cortex can be stimulated (10 Hz 3000-4000 pulses) (AFTANAS) stimulation of the supplementary motor cortex may improve diaphragm function.

\section{Transcranial direct current stimulation}

Anodal transcranial direct current stimulation (EDCS) has been used to increase vagus nerve activity employing bifrontal positioning of electrodes or coils [96]. Temporal cortex autonomic modulation with reduced sympathetic activity, increased baroreceptor sensitivity and a reduced heart rate at submaximal exercise [97] has been reported.

\section{Clinical trials}

Autonomic dysfunction is common in PASC, causing symptoms that impair quality of life, daily activities and return to work. Supportive measures as previously summarized are a mainstay of management, but many patients remain symptomatic. Accordingly, clinical trials with clear endpoints, reproducibility and validation are needed. Areas of investigation and treatment modalities should include $\beta$-blockers (e.g. nebivolol versus ivabradine) on a background of standard therapy with endpoints of dyspnea index, pulmonary function parameters, heart rate at rest and in response to activity and quality of life; extracranial stimulation versus standard of care with endpoints of functional status, fatigue index and quality of life; mineralocorticoid inhibition versus standard of care for nocturnal hypertension; and graded supine exercise training and mechanical breathing exercise program versus standard care for post-exercise exhaustion. These a just a few examples.

\section{Summary}

SARS-CoV-2 is the cause of COVID-19 and is responsible for 175,541,600 confirmed cases and 3,798,361 deaths worldwide. There are upward of 220,000 cases daily (www. who.com; accessed 14 June 2021). Most persons who 
contract COVID-19 survive their illness, but up to $70 \%$ of survivors have one or more symptoms lasting for 3 months or greater. Resting tachycardia, dyspnea, post-exercise exhaustion, sleep disturbance and fatigue are common, lingering symptoms - each could be explained by post-viral autonomic dysfunction. Comprehensive testing in experienced centers and clinical trials are needed to address a common barrier to full recovery in persons surviving COVID-19.

\section{References}

1. Goldberger JJ et al (2019) Autonomic nervous system dysfunction: JACC focus seminar. J Am Coll Cardiol 73(10):1189-1206

2. Del Rio R, Marcus NJ, Inestrosa NC (2020) Potential role of autonomic dysfunction in Covid-19 morbidity and mortality. Front Physiol 11:561749

3. Tracey KJ (2002) The inflammatory reflex. Nature 420(6917):853-859

4. Koopman FA et al (2011) Restoring the balance of the autonomic nervous system as an innovative approach to the treatment of rheumatoid arthritis. Mol Med 17(9-10):937-948

5. Armour JA et al (1997) Gross and microscopic anatomy of the human intrinsic cardiac nervous system. Anat Rec 247(2):289-298

6. Chouchou F, Desseilles M (2014) Heart rate variability: a tool to explore the sleeping brain? Front Neurosci 8:402

7. Guyenet PG (2006) The sympathetic control of blood pressure. Nat Rev Neurosci 7(5):335-346

8. Reisert M, Weiller C, Hosp JA (2021) Displaying the autonomic processing network in humans-a global tractography approach. Neuroimage 231:117852

9. Shivkumar K et al (2016) Clinical neurocardiology defining the value of neuroscience-based cardiovascular therapeutics. J Physiol 594(14):3911-3954

10. Isaacson SH et al (2021) Management strategies for comorbid supine hypertension in patients with neurogenic orthostatic hypotension. Curr Neurol Neurosci Rep 21(4):18

11. Becker RC (2020) COVID-19-associated vasculitis and vasculopathy. J Thromb Thrombolysis 50:499-511

12. Robles-Cabrera A et al (2014) The cardiovagal, cardiosympathetic and vasosympathetic arterial baroreflexes and the neural control of short-term blood pressure. Rev Neurol 59(11):508-516

13. Kirchheim HR (1976) Systemic arterial baroreceptor reflexes. Physiol Rev 56(1):100-177

14. Mortara A et al (1997) Arterial baroreflex modulation of heart rate in chronic heart failure: clinical and hemodynamic correlates and prognostic implications. Circulation 96(10):3450-3458

15. Grönloh MLB, Arts JJG, van Buul JD (2021) Neutrophil transendothelial migration hotspots - mechanisms and implications. J Cell Sci. https://doi.org/10.1242/jcs.255653

16. Dessalles CA, Babataheri A, Barakat AI (2021) Pericyte mechanics and mechanobiology. J Cell Sci. https://doi.org/10.1242/jcs. 240226

17. Shepro D, Morel NML (1993) Pericyte physiology. FASEB J 7(11):1031-1038

18. Sims DE (2000) Diversity within pericytes. Clin Exp Pharmacol Physiol 27(10):842-846

19. O'Rourke MF, Safar ME (2005) Relationship between aortic stiffening and microvascular disease in brain and kidney: cause and logic of therapy. Hypertension 46(1):200-204
20. Proebstl D et al (2012) Pericytes support neutrophil subendothelial cell crawling and breaching of venular walls in vivo. J Exp Med 209(6):1219-1234

21. Bohannon DG, Long D, Kim WK (2021) Understanding the heterogeneity of human pericyte subsets in blood-brain barrier homeostasis and neurological diseases. Cells 10(4):890

22. Bruns RR, Palade GE (1968) Studies on blood capillaries: I. General organization of blood capillaries in muscle. J Cell Biol 37(2):244-276

23. Dore-Duffy P et al (2000) Pericyte migration from the vascular wall in response to traumatic brain injury. Microvasc Res 60(1):55-69

24. Litviňuková M et al (2020) Cells of the adult human heart. Nature 588(7838):466-472

25. Muus C et al (2021) Single-cell meta-analysis of SARSCoV-2 entry genes across tissues and demographics. Nat Med 27(3):546-559

26. Behrens R et al (2021) Mini review: central organization of airway afferent nerve circuits. Neurosci Lett 744:135604

27. Driessen AK et al (2018) Reflex regulation of breathing by the paratrigeminal nucleus via multiple bulbar circuits. Brain Struct Funct 223(9):4005-4022

28. Silva ÉP et al (2021) Heart rate recovery in asthmatic children and adolescents after clinical field test. BMC Pulm Med 21(1):61

29. Tang LYW et al (2021) Autonomic alterations after pulmonary vein isolation in the CIRCA-DOSE (cryoballoon vs irrigated radiofrequency catheter ablation) study. J Am Heart Assoc 10(5): 18610

30. García-Lunar I et al (2020) Neurohormonal modulation as a therapeutic target in pulmonary hypertension. Cells 9(11):2521

31. Constantine A, Dimopoulos K (2021) Pulmonary artery denervation for pulmonary arterial hypertension. Trends Cardiovasc Med 31(4):252-260

32. Perros F et al (2015) Nebivolol for improving endothelial dysfunction, pulmonary vascular remodeling, and right heart function in pulmonary hypertension. J Am Coll Cardiol 65(7):668-680

33. Tantucci C et al (2021) Respiratory function, autonomic dysfunction, and systemic inflammation are closely linked in patients with COPD and tidal flow limitation: An exploratory study. Respir Physiol Neurobiol 284:103565

34. Heindl $\mathrm{S}$ et al (2001) Marked sympathetic activation in patients with chronic respiratory failure. Am J Respir Crit Care Med 164(4):597-601

35. Guardiola J, Saad M, Yu J (2019) Hypertonic saline stimulates vagal afferents that respond to lung deflation. Am J Physiol 317(6):R814-R817

36. Koike H (2015) Acute sensory neuropathies and acute autonomic neuropathies. Brain Nerve 67(11):1377-1387

37. Feldman EL et al (1991) Acute pandysautonomic neuropathy. Neurology 41(5):746-748

38. Reilly CC et al (2020) Breathlessness and dysfunctional breathing in patients with postural orthostatic tachycardia syndrome (POTS): the impact of a physiotherapy intervention. Auton Neurosci 223:102601

39. Tooba R et al (2021) Dyspnea in chronic low ventricular preload states. Ann Am Thorac Soc 18(4):573-581

40. Cherneva RV, Denchev SV, Cherneva ZV (2020) The link between dynamic hyperinflation, autonomic dysfunction and exercise testing parameters with masked heart failure in patients with nonsevere obstructive pulmonary disease. J Basic Clin Physiol Pharmacol 32(3): 179-188

41. Cerri M, Amici R (2021) Thermoregulation and sleep: functional interaction and central nervous control. Compr Physiol 11(2):1591-1604 
42. Liang $\mathbf{J}$ et al (2018) The independent and combined effects of respiratory events and cortical arousals on the autonomic nervous system across sleep stages. Sleep Breath 22(4):1161-1168

43. Monti A et al (2002) Autonomic control of the cardiovascular system during sleep in normal subjects. Eur J Appl Physiol 87(2):174-181

44. Kai $\mathrm{S}$ et al (2021) Cardiac autonomic nervous system activity during slow breathing in supine position. Rehabil Res Pract 2021:6619571

45. Kai $\mathrm{S}$ et al (2016) Effectiveness of moderate intensity interval training as an index of autonomic nervous activity. Rehabil Res Pract 2016:6209671

46. Delgado-Ortiz L et al (2021) Physical activity and cardiac autonomic dysfunction in patients with chronic obstructive pulmonary disease: a cross-sectional analysis. Ann Phys Rehabil Med. https:// doi.org/10.1016/j.rehab.2021.101501

47. Stockelman KA et al (2021) Regular aerobic exercise counteracts endothelial vasomotor dysfunction associated with insufficient sleep. Am J Physiol Heart Circ Physiol 320(3):H1080-h1088

48. Wirth KJ, Scheibenbogen C (2021) Pathophysiology of skeletal muscle disturbances in Myalgic Encephalomyelitis/Chronic Fatigue Syndrome (ME/CFS). J Transl Med 19(1):162

49. Stute NL, Koopmans PJ (2021) Pushing the needle forward on the relationship between autoimmunity and autonomic dysfunction. J Physiol 599(4):1039-1040

50. Feldman JL, Gray PA (2000) Sighs and gasps in a dish. Nat Neurosci 3(6):531-532

51. Porges WL et al (2000) Heart-lung interactions: the sigh and autonomic control in the bronchial and coronary circulations. Clin Exp Pharmacol Physiol 27(12):1022-1027

52. Bowes $\mathrm{G}$ et al (1983) Carotid chemoreceptor regulation of expiratory duration. J Appl Physiol Respir Environ Exerc Physiol 54(5):1195-1201

53. Wilhelm FH, Trabert W, Roth WT (2001) Physiologic instability in panic disorder and generalized anxiety disorder. Biol Psychiatry 49(7):596-605

54. Boulding R et al (2016) Dysfunctional breathing: a review of the literature and proposal for classification. Eur Respir Rev 25(141):287-294

55. Courtney R et al (2011) Medically unexplained dyspnea: partly moderated by dysfunctional (thoracic dominant) breathing pattern. J Asthma 48(3):259-265

56. Debeaumont $\mathrm{D}$ et al (2021) Cardiopulmonary exercise testing to assess persistent symptoms at 6 months in people with COVID19 who survived hospitalization - a pilot study. Phys Ther 101:6. https://doi.org/10.1093/ptj/pzab099

57. Bajić D, Đajić V, Milovanović B (2021) Entropy analysis of COVID-19 cardiovascular signals. Entropy (Basel) 23(1):87

58. Zou R et al (2021) Clinical characteristics and hemodynamic responses to head-up tilt test in children and adolescents with unexplained sighing. Neurol Sci. https://doi.org/10.1007/ s10072-020-04956-8

59. Denis F et al (2021) A self-assessment web-based app to assess trends of the COVID-19 pandemic in France: observational study. J Med Internet Res 23(3):e26182

60. Quer $\mathrm{G}$ et al (2021) Wearable sensor data and self-reported symptoms for COVID-19 detection. Nat Med 27(1):73-77

61. Zens M et al (2020) App-based tracking of self-reported COVID19 symptoms: analysis of questionnaire data. J Med Internet Res 22(9):21956

62. Johansson M et al (2021) Long-haul post-COVID-19 symptoms presenting as a variant of postural orthostatic tachycardia syndrome: the Swedish experience. JACC Case Rep 3(4):573-580

63. Mondal R et al (2021) COVID-19 and emerging spinal cord complications: a systematic review. Mult Scler Relat Disord $51: 102917$
64. Garg RK, Paliwal VK, Gupta A (2021) Spinal cord involvement in COVID-19: a review. J Spinal Cord Med. https://doi.org/10.1080/ 10790268.2021.1888022

65. Su XW et al (2020) SARS-CoV-2-associated Guillain-Barré syndrome with dysautonomia. Muscle Nerve 62(2):E48-e49

66. Andalib $S$ et al (2021) Peripheral nervous system manifestations associated with COVID-19. Curr Neurol Neurosci Rep 21(3):9

67. Watari $\mathrm{M}$ et al (2018) Autoimmune postural orthostatic tachycardia syndrome. Ann Clin Transl Neurol 5(4):486-492

68. Raj SR et al (2009) Propranolol decreases tachycardia and improves symptoms in the postural tachycardia syndrome: less is more. Circulation 120(9):725-734

69. Olshansky B, Sullivan RM (2019) Inappropriate sinus tachycardia. Europace 21(2):194-207

70. Baruscotti $\mathrm{M}$ et al (2016) Current understanding of the pathophysiological mechanisms responsible for inappropriate sinus tachycardia: role of the If "funny" current. J Interv Card Electrophysiol 46(1):19-28

71. Calò L et al (2010) Efficacy of ivabradine administration in patients affected by inappropriate sinus tachycardia. Heart Rhythm 7(9):1318-1323

72. Ptaszynski P et al (2013) Metoprolol succinate vs. ivabradine in the treatment of inappropriate sinus tachycardia in patients unresponsive to previous pharmacological therapy. Europace 15(1):116-121

73. Mathew ST, Po SS, Thadani U (2018) Inappropriate sinus tachycardia-symptom and heart rate reduction with ivabradine: a pooled analysis of prospective studies. Heart Rhythm 15(2):240-247

74. Engin AB, Engin ED, Engin A (2021) Current opinion in neurological manifestations of SARS-CoV-2 infection. Curr Opin Toxicol 25:49-56

75. Paniz-Mondolfi A et al (2020) Central nervous system involvement by severe acute respiratory syndrome coronavirus-2 (SARSCoV-2). J Med Virol 92(7):699-702

76. Rodriguez M et al (2020) Impact of Severe Acute Respiratory Syndrome Coronavirus 2 (SARS-CoV-2) in the nervous system: implications of COVID-19 in neurodegeneration. Front Neurol 11:15. https://doi.org/10.3389/fneur.2020.583459

77. Kanberg N et al (2020) Neurochemical evidence of astrocytic and neuronal injury commonly found in COVID-19. Neurology 95(12): $1754-\mathrm{e} 1759$

78. Zhou $Z$ et al (2020) Understanding the neurotropic characteristics of SARS-CoV-2: from neurological manifestations of COVID-19 to potential neurotropic mechanisms. J Neurol 267(8):2179-2184

79. Becker RC (2020) COVID-19-associated vasculitis and vasculopathy. J Thromb Thrombolysis 50(3):499-511

80. Meinhardt J et al (2021) Olfactory transmucosal SARS-CoV-2 invasion as a port of central nervous system entry in individuals with COVID-19. Nat Neurosci 24(2):168-175

81. Al-Dalahmah O et al (2020) Neuronophagia and microglial nodules in a SARS-CoV-2 patient with cerebellar hemorrhage. Acta Neuropathol Commun 8(1):147

82. Boldrini M, Canoll PD, Klein RS (2021) How COVID-19 affects the brain. JAMA Psychiatry 78:682

83. Blitshteyn S, Whitelaw S (2021) Postural orthostatic tachycardia syndrome (POTS) and other autonomic disorders after COVID-19 infection: a case series of 20 patients. Immunol Res 69:212

84. Tagaloa E et al (2021) A rare case of Guillain-Barré syndrome with severe pandysautonomia. J Investig Med High Impact Case Rep 9:23247096211019560

85. Kaliyaperumal D et al (2021) Characterization of cardiac autonomic function in COVID-19 using heart rate variability: a hospital based preliminary observational study. J Basic Clin Physiol Pharmacol 32:247 
86. Guaraldi $P$ et al (2020) Testing cardiovascular autonomic function in the COVID-19 era: lessons from Bologna's Autonomic Unit. Clin Auton Res 30(4):325-330

87. Haensel A et al (2008) The relationship between heart rate variability and inflammatory markers in cardiovascular diseases. Psychoneuroendocrinology 33(10):1305-1312

88. Haarala A et al (2011) Heart rate variability is independently associated with C-reactive protein but not with Serum amyloid A. The cardiovascular risk in young finns study. Eur J Clin Invest 41(9):951-957

89. Martínez-Rosales E et al (2020) Heart rate variability in women with systemic lupus erythematosus: association with healthrelated parameters and effects of aerobic exercise. Int J Environ Res Public Health 17(24):9501

90. Baptista AF et al (2020) Applications of non-invasive neuromodulation for the management of disorders related to COVID-19. Front Neurol 11:573718

91. Moroi MK et al (2021) Prevention and management of supine hypertension in patients with orthostatic hypotension. Am J Ther 28(2):e228-e231
92. Kario K et al (2021) Effect of the nonsteroidal mineralocorticoid receptor blocker, esaxerenone, on nocturnal hypertension: a post hoc analysis of the ESAX-HTN study. Am J Hypertens 34(5):540-551

93. Okamoto LE et al (2021) Local passive heat for the treatment of hypertension in autonomic failure. J Am Heart Assoc 10(7):18979

94. Shannon J et al (1997) The hypertension of autonomic failure and its treatment. Hypertension 30(5):1062-1067

95. Lo A et al (2021) Pharmacokinetics and pharmacodynamics of ampreloxetine, a novel, selective norepinephrine reuptake inhibitor, in symptomatic neurogenic orthostatic hypotension. Clin Auton Res 31:395-403

96. Nikolin $\mathrm{S}$ et al (2019) Comparison of site localization techniques for brain stimulation. J ECT 35(2):127-132

97. Okano AH et al (2015) Brain stimulation modulates the autonomic nervous system, rating of perceived exertion and performance during maximal exercise. Br J Sports Med 49(18):1213-1218

Publisher's Note Springer Nature remains neutral with regard to jurisdictional claims in published maps and institutional affiliations. 